\title{
UGV Trailer Position Estimation Using a Dynamic Base RTK System
}

\author{
William E. Travis* and David W. Hodo ${ }^{\dagger}$ and David M. Bevly ${ }^{\ddagger}$ and John Y. Hung ${ }^{\S}$ \\ Auburn University, Auburn, AL, 36832, USA
}

\begin{abstract}
In this paper, an algorithm for a dynamic base real-time kinematic (DRTK) GPS is presented and a novel application to the guidance and control of a mobile robot pulling a trailer is discussed. The DRTK system is used to estimate the relative position between the robot and trailer in the autonomous system described, and the results are compared to traditional methods utilizing an optical encoder. Geometry error is analytically predicted and the influence on trailer position is shown. Experimental results show a marginal accuracy improvement over a properly calibrated encoder based system but a significant improvement when calibration errors are present.
\end{abstract}

\section{Introduction}

The control of mobile robot-trailer systems has been researched extensively. ${ }^{1-3}$ A good overview of the problem is given by De Luca. ${ }^{4}$ The majority of research in the area focuses on controlling the position of the robot, while keeping the trailer dynamics stable. For some applications, however, the placement of the trailer is more important than that of the robot. One such application is geophysical surveys for detection of electromagnetic anomalies.

A robotic tractor-trailer system is currently being developed at Auburn University in conjunction with the U.S. Army Corps of Engineers and the Environmental Security Technology Certification Program (ESTCP) for the purpose of locating and mapping unexploded ordnance (UXO). It consists of a four-wheel differential drive robot (Segway RMP) towing a trailer, which carries various geophysical equipment such as magnetometers and electromagnetic sensors. The robot autonomously tows the trailer along a predefined path to map a given area. A picture of the robot towing a Geonics EM61-MK2 electromagnetic sensor is shown in figure 1. A detailed description of the system can be found in previous works by the authors. ${ }^{5}$

Precise control of the position of the trailer is required in order to create an accurate geophysical map. A control algorithm has been designed for the robot to guide the trailer along a path consisting of lines and circular arcs. For this algorithm to perform properly, however, it requires precise knowledge of the trailer's current position. Currently, the position of the trailer is calculated from the position and orientation of the robot and the angle between the robot and trailer.

Another option for determining the trailer's position is to measure it directly using a real time kinematic (RTK) GPS system. RTK techniques take advantage of the spatial and temporal error correlation in the GPS signal to produce an accurate relative position, or baseline, between two points. Traditionally, an RTK system involves a minimum of two GPS receivers; one is statically stationed at a known location, and the other is allowed to roam. The relative position vector is then combined with the known location of the base to provide a global position that is accurate to less than two centimeters. An RTK system is relativey expensive, however, and requires that a base station be placed on a known location.

A dynamic base RTK (or DRTK) algorithm has been developed that removes the constraint of the static base while retaining the high accuracy relative position estimate. Similar algorithms have been developed in the past for formation flight, automated flight refueling, and automated shipboard landing. ${ }^{6-8}$ When applied to the system detailed above, the trailer's position relative to the robot can be estimated using the DRTK

\footnotetext{
${ }^{*}$ Graduate Research Assistant, Mechanical Engineering, 270 Ross Hall, Student Member.

${ }^{\dagger}$ Graduate Research Assistant, Electrical and Computer Engineering, 201 Broun Hall

$¥$ Associate Professor, Mechanical Engineering, 270 Ross Hall

$\S$ Professor, Electrical and Computer Engineering, 201 Broun Hall
} 
method. It will be shown that the accuracy of this position vector can lead to improved performance of the trailer control algorithm.

In this paper, a description of the DRTK algorithm will be given. Methods will be described that allow the position of the trailer to be determined using both an encoder and the DRTK algorithm. Experiments are carried out using both methods and the accuracies are compared. Results show a marginal accuracy improvement over a properly calibrated encoder based method, but a significant improvement is seen when calibration errors are present.

\section{Trailer Position Estimation Using an Encoder}

\section{II.A. Description of the Current Method}

Currently, the position of the robot is determined using a GPS/INS positioning system. The positioning system consists of a NovAtel DL4-Plus GPS receiver blended with a HG1700 IMU using the NovAtel SPAN system. The GPS receiver operates in RTK mode, receiving corrections from a static base station at a known location. The relative angle between the robot and the trailer (hitch angle) is measured using an optical encoder. The geometry of the system is known, so the position of the trailer can then be calculated from the position of the robot and the hitch angle using:

$$
\begin{aligned}
e_{t} & =e_{r}-l_{r} \sin \left(\psi_{r}\right)-l_{t} \sin \left(\psi_{r}+\varphi\right) \\
n_{t} & =n_{r}-l_{r} \cos \left(\psi_{r}\right)-l_{t} \cos \left(\psi_{r}+\varphi\right) \\
\psi_{t} & =\psi_{r}+\varphi
\end{aligned}
$$

where $\left(e_{r}, n_{r}\right)$ and $\left(e_{t}, n_{t}\right)$ are the positions of the robot and trailer in UTM coordinates, $\psi_{r}$ and $\psi_{t}$ are the orientations of the robot and trailer respectively measured clockwise with respect to north, and $\varphi$ is the hitch angle. A schematic representation of the system is given in figure 2 .

Although the position and orientation of the robot are currently determined using a GPS/INS system with RTK corrections, that is not the focus of this work. Only the accuracies in the relative measurement between the robot and trailer are examined. An accurate measurement of the robot's global position and heading are assumed but could come from GPS or other methods such as odometry, vision systems, etc.

\section{II.B. Limitations of the Current Method}

Using an encoder to determine the position of a trailer towed behind a robot is a commonly used technique. $^{9-12}$ It can, however, introduce errors that, while not an issue in some applications, can cause a significant problem if the precise location of the trailer is desired. It was assumed earlier that the geometry of the system is known. This requires exact measurements of the hitch and trailer lengths $\left(l_{r}\right.$ and $\left.l_{t}\right)$. Errors in measuring these lengths result in an inaccurate estimate of the trailer's position. An accurate robot heading and hitch angle measurement are also required for this method. One common source of error is a bias

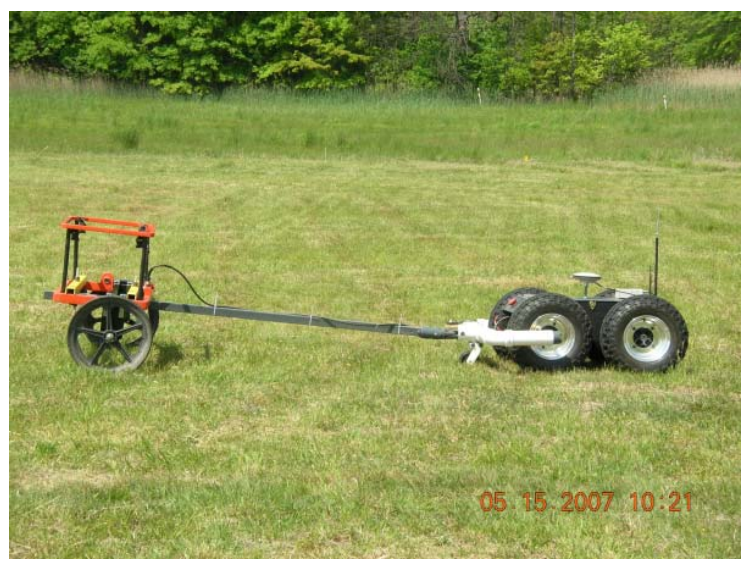

Figure 1. Robot and Trailer 


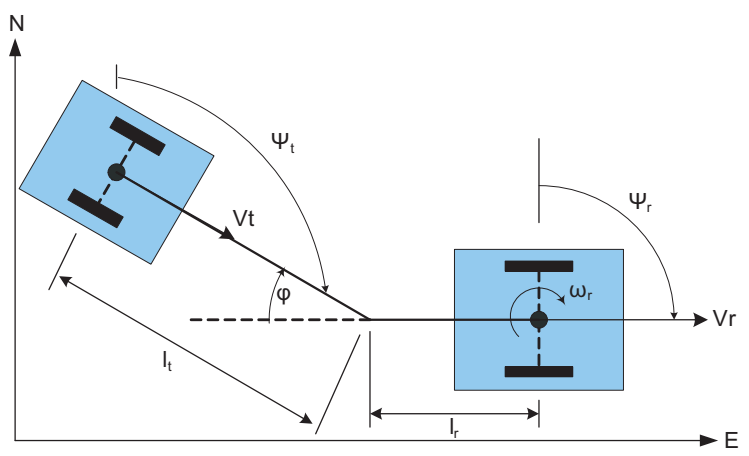

Figure 2. Robot - Trailer System Model

in the encoder measurement, which can result from improper alignment, joint backlashes, etc. The effects of these errors are studied extensively in previous works by the authors. ${ }^{13,14}$

Other more fundamental problems with this method can also affect the accuracy of the trailer position. The hitch connecting the robot and trailer has three degrees of freedom (roll, pitch, and yaw), but only one angle (yaw) is measured. When the trailer's position is calculated, it is assumed that the robot and trailer are on smooth ground and therefore in the same plane. The majority of experimental results given in the literature focus on vehicles that operate on streets or in indoor environments so the assumption is generally valid. On more varied terrain, which would be expected when performing outdoor surveys, the assumption does not always hold. The hitch could be modified to allow all three degrees of freedom to be measured but would require a more complex and expensive hitch and sensing system.

\section{Trailer Position Estimation Using DRTK}

\section{III.A. DRTK Algorithm}

The DTRK algorithm operates in principle like a traditional RTK algorithm; errors in the range and carrier phase measurements which are correlated in time and across space are differenced out using multiple receivers to determine the relative position between those receivers. For high accuracy applications, the carrier phase measurements are used due to their superior accuracy over the pseudorange measurements. Typical accuracies for pseudorange measurements are half a meter, while accuracies for the phase measurement are around five millimeters. ${ }^{15}$ The phase measurement is also more robust to multipath error. ${ }^{16}$ In order to use the phase measurements to obtain higher accuracy relative position estimates, the integer number of cycles between the user and satellite, or integer ambiguity, must be resolved.

Mathematical models of the signals as defined by Misra and Enge ${ }^{15}$ are given in Eqs. 2 and 3:

$$
\begin{gathered}
\rho_{A}^{j}(t)=u_{A}^{j} \vec{r}_{A}^{j}(t)+\gamma(t)+\xi(t)+c\left(\delta t_{A}(t)-\delta t^{j}(t)\right)+\epsilon_{\rho}(t) \\
\phi_{A}^{j}(t)=u_{A}^{j} \vec{r}_{A}^{j}(t)-\gamma(t)+\xi(t)+\lambda a_{A}^{j}(t)+c\left(\delta t_{A}(t)-\delta t^{j}(t)\right)+\epsilon_{\phi}(t)
\end{gathered}
$$


The variables are defined as follows:

$\rho_{A}^{j} \quad$ the measured range (pseudorange), in meters, from receiver $A$ to satellite $j$

$\phi_{A}^{j} \quad$ the measured carrier signal phase, in meters, from receiver $A$ to satellite $j$

$u_{r}^{j} \quad$ the unit vector from receiver $\mathrm{r}$ to satellite $\mathrm{j}$

$\vec{r}_{A}^{j} \quad$ the true range vector, in meters, from receiver $A$ to satellite $j$

$\gamma \quad$ the ionospheric delay/advancement, in meters

$\xi \quad$ the tropospheric delay, in meters

$\delta t_{A}$ the clock error, in seconds, at the receiver

$\delta t^{j} \quad$ the clock error, in seconds, at satellite $j$

$\lambda \quad$ the wavelength of the carrier signal, in meters

$a_{A}^{j} \quad$ the integer number of cycles from receiver $A$ to satellite $j$

$c \quad$ the speed of light, in meters per second

$\epsilon \quad$ system noise, including multipath

Most GPS receivers use a code modulated onto the carrier to determine the range between the user and satellite. This range measurement is given often referred to as a pseudorange due to the errors present in the measurement. The error due to atmospheric disturbances and satellite clock inaccuracies in the respective signals can be reduced to negligible quantities if two receivers are in close proximity $(<20 \mathrm{~km})$. A single difference for the carrier measurement is shown below:

$$
\begin{aligned}
\Delta \phi_{A B}^{j}(t) & =\phi_{B}^{j}(t)-\phi_{A}^{j}(t) \\
& =\left(u_{B}^{j}-u_{A}^{j}\right) \vec{r}_{A B}^{j}(t)+\lambda \Delta a_{A B}^{j}+c\left(\delta t_{B}(t)-\delta t_{A}(t)\right)+\epsilon_{\Delta \phi}(t)
\end{aligned}
$$

Notice the atmospheric and satellite clock errors have been removed, but the noise has been increased. It is important to note that high atmospheric error residuals can exist in some situations, such as high ionospheric activity or severe weather, and additional techniques must be used to reduce the errors. ${ }^{17}$

A single differenced pseudorange can be expressed in the same manner, although the ambiguity term is no longer present.

$$
\begin{aligned}
\Delta \rho_{A B}^{j}(t) & =\rho_{B}^{j}(t)-\rho_{A}^{j}(t) \\
& =\left(u_{B}^{j}-u_{A}^{j}\right) \vec{r}_{A B}^{j}(t)+c\left(\delta t_{B}(t)-\delta t_{A}(t)\right)+\epsilon_{\Delta \rho}(t)
\end{aligned}
$$

In the single differenced equations, the remaining error sources are the receiver clock biases and noise. An integer ambiguity is present in the carrier phase equations. A double difference is formed by subtracting the single differences from a "base" single difference (denoted by satellite $\mathrm{z}$ ). The base satellite is chosen as the one closest to the zenith, as the signal from this satellite travels the most direct route to the receiver and is theoretically subjected to fewer sources of interference. The double differenced carrier phase and pseudorange expressions are listed in Eqs. 6 and 7.

$$
\begin{aligned}
\nabla \Delta \phi_{A B}^{j z}(t) & =\Delta \phi_{A B}^{z}(t)-\Delta \phi_{A B}^{j}(t) \\
& =\left(\Delta u_{A B}^{z}-\Delta u_{A B}^{j}\right) \vec{r}_{A B}^{j z}(t)+\lambda \nabla \Delta a_{A B}^{j z}+\epsilon_{\nabla \Delta \phi}(t) \\
\nabla \Delta \rho_{A B}^{j z}(t) & =\Delta \rho_{A B}^{z}(t)-\Delta \rho_{A B}^{j}(t) \\
& =\left(\Delta u_{A B}^{z}-\Delta u_{A B}^{j}\right) \vec{r}_{A B}^{j z}(t)+\epsilon_{\nabla \Delta \rho}(t)
\end{aligned}
$$

The double differenced measurements can be expressed in matrix form using Eq. 8,

$$
\left[\begin{array}{l}
\nabla \Delta \rho(t) \\
\nabla \Delta \phi(t)
\end{array}\right]=G \vec{r}_{A B}(t)+H \nabla \Delta a
$$


where

$$
\begin{gathered}
G=\left[\begin{array}{c}
\nabla \Delta u \\
\nabla \Delta u \\
\nabla \Delta u
\end{array}\right] \\
H=\left[\begin{array}{c}
0 \\
\lambda I
\end{array}\right]
\end{gathered}
$$

The subscript $A B$ has been removed from all variables except for the relative range for simplicity. Note this equation can be expanded to contain double differenced pseudoranges, phases, and ambiguities of multiple carrier frequencies if desired. However, the focus of this work is on a solution that utilizes only the L1 signal in order to utilize low cost receivers and keep production costs at a minumum.

Precise resolution of the integer ambiguities is fundamental to the accurate estimation of a relative position vector. Equation 8 can be used in a two stage estimation scheme where the ambiguities are estimated and subsequently fixed, and then used to estimate the relative position vector. The position states are irrelevant to the estimation of the ambiguities, and can be removed by determining the left-null space, $L$, of the geometry matrix, $G$, from Eq. 9 such that $L G=0$. This new equation, shown below, can be used to estimate the ambiguities in a discrete, linear Kalman filter. ${ }^{18}$

$$
L\left[\begin{array}{c}
\nabla \Delta \rho(t) \\
\nabla \Delta \phi(t)
\end{array}\right]=L H \nabla \Delta a
$$

Measurement noise on the individual signals was modeled as thermal noise jitter in the receiver tracking loops. This incorporated the carrier to noise ratio, $C / N_{0}$, so the degraded signals were de-weighted. A third order loop filter was assumed with a bandwidth, $B_{n}$, of $18 \mathrm{~Hz}$ and a predetection integration time, $T_{s}$, of five milliseconds. The correlator loop spacing, $d$, was half a chip. The reasoning for the selection of these values is thoroughly explained in. ${ }^{19}$

$$
\begin{gathered}
\sigma_{\epsilon_{\phi}}=\frac{\lambda}{2 \pi} \sqrt{\frac{B_{n}}{C / N_{0}}\left(1+\frac{1}{T_{s} C / N_{0}}\right)} \\
\sigma_{\epsilon_{\rho}}=\lambda \sqrt{\frac{4 d^{2} B_{n}}{C / N_{0}}\left(2(1-d)+\frac{4 d}{T_{s} C / N_{0}}\right)}
\end{gathered}
$$

A covariance matrix for the undifferenced signals can be constructed as follows:

$$
R=\left[\begin{array}{cc}
\sigma_{\epsilon_{\rho}}^{2} I & 0 \\
0 & \sigma_{\epsilon_{\phi}}^{2} I
\end{array}\right]
$$

Assuming the carrier phase and pseudorange measurements are linearly independent, the covariance of the single differenced measurements can be derived as seen in. ${ }^{16}$ A matrix is constructed that converts a vector of undifferenced signals to a vector of single differenced signals $\left(C_{S D}\right)$. This matrix is used to transform the undifferenced covariance matrix to a covariance matrix for the single differenced signals.

$$
R_{\Delta}=E\left[(\Delta[\cdot](t)-\overline{\Delta[\cdot]}(t))^{2}\right]=C_{S D} R C_{S D}^{T}
$$

A similar approach can be used to determine the covariance matrix of double differenced signals to be used in the Kalman filter. A matrix is constructed that converts a vector of single differenced measurements to a vector of double differenced measurements $\left(C_{D D}\right)$. This is now used to transform the covariance matrix in Eq. 15 to a covariance matrix for the double differenced measurements.

$$
R_{\nabla \Delta}=E\left[(\nabla \Delta[\cdot](t)-\overline{\nabla \Delta[\cdot]}(t))^{2}\right]=C_{D D} R_{\Delta} C_{D D}^{T}
$$

The state transition matrix, $\Phi$, is simply identity since the position states were removed by multipling the measurement vector and matrix by the left-null of the geometry matrix. The process noise covariance matrix is tuned such that the steady state estimated covariance from the Kalman filter reaches a value between 1/4 and $1 / 5$ of a wavelength. 
Given enough satellites, the Kalman filter, as described, will estimate a non-integer number of carrier cycles. It is crucial to obtain integer vaules for the cycle ambiguity if a high precision relative position vector is desired. The LAMBDA method ${ }^{20}$ has been proven to provide the highest probability of aquiring the correct set of integer ambiguities among many integer ambiguity acquisition algorithms. ${ }^{21}$ The floating ambiguities and their associated estimated covariance from the Kalman filter are input into the LAMBDA algorithm. It decorrelates the ambiguities to produce a minimized search space and outputs the possible solution sets $(\tilde{a})$ contained within that space with their covariance matrices $\left(P_{\tilde{a}}\right)$ ranked from best to worst. ${ }^{22}$ A common method to determine the correct ambiguity set is to calculate a ratio between the square norms of the integer ambiguities, where the best set is deemed correct if the ratio is above some value, $\kappa \cdot{ }^{6,23}$ In the equation below, a ratio of the best set to the second best set is calculated. The value for $\kappa$ is selected by the user and is generally 1.5 or 2 .

$$
\frac{\left[\hat{a}-\tilde{a_{2}}\right]^{T} P_{\tilde{a_{2}}}^{-1}\left[\hat{a}-\tilde{a_{2}}\right]}{\left[\hat{a}-\tilde{a_{1}}\right]^{T} P_{\tilde{a_{1}}}^{-1}\left[\hat{a}-\tilde{a_{1}}\right]} \underset{\text { reject }}{\stackrel{a c c e p t}{\gtrless}} \kappa
$$

Once the integer ambiguities have been correctly determined, a least squares procedure is used to determine a precise relative position vector. The measurement vector consists of only the double differenced carrier measurements with the ambiguity removed. The geometry matrix, $G$ is compensated to reflect the reduced size of the measurement vector.

$$
\vec{r}_{A B}=\left[G^{T} G\right]^{-1} G^{T}[\nabla \Delta \phi-\tilde{a}]
$$

\section{III.B. Estimating Trailer Position}

The hitch angle $(\varphi)$ and robot heading $\left(\psi_{r}\right)$ measurements are not needed if the DRTK method is used to estimate the trailer position. Instead, a second GPS antenna is placed on the trailer. A baseline is estimated in the ECEF frame from the robot antenna to the trailer antenna using the DRTK algorithm. The trailer's position $\vec{r}_{B}$ is then determined by simply adding the baseline $\vec{r}_{A B}$ to the robot's ECEF position $\vec{r}_{A}$.

$$
\vec{r}_{B}=\vec{r}_{A}+\vec{r}_{A B}
$$

Once the trailer's ECEF position is known, it can be translated into different coordinate frames that are more suitable for ground vehicle control. For this work, the resulting trailer positions were translated into the UTM frame for use by the controller.

\section{III.C. Limitations of the DRTK Method}

The DRTK method requires a sufficient number of GPS satellites that are visible to both receivers. Any significant obstruction or interference can hinder the DRTK algorithm if enough satellites cannot be seen by the receivers. In some environments, such as forests, cities, or canyons, this could be an issue as GPS availability is limited. Integration of the algorithm with other sensors could improve its robustness by providing a lower precision baseline estimate during the outage, thus reducing the integer ambiguity acquisition time when the GPS signal is visible again.

Another drawback to the DRTK method is cost. Usually, higher end GPS receivers are required to perform the computations necessary for an RTK system. These systems cost between $\$ 2,000$ and $\$ 15,000$ USD, where an encoder system can cost around \$200 USD. To address this financial issue, future work will produce a DRTK system using low cost L1 GPS receivers and a few small microprocessors, with a target production price of $\$ 200$ USD per receiver.

\section{Trailer Position Estimation Comparison}

\section{IV.A. Experimental Setup}

The accuracy of the trailer position estimate using the encoder based method described in Sec. II and the DRTK based method described in Sec. III were compared experimentally. A path consisting of several straight line segments connected by circular arcs in an S-type pattern was driven autonomously for the experiments. Two NovAtel Propak V-3 receivers were used to log the range measurements, ephemerides, 
and user position, which are needed by the DRTK algorithm. One was placed on the robot, and the other was placed on the trailer. The signal from the antenna on the robot was split using a GPS Networking Inc. splitter, so the DL4-Plus and PropakV-3 received the same signal. A Navcom 2050M was set up as a static RTK base station, and the corrections were broadcast via $900 \mathrm{MHz}$ radio modems to the robot mounted DL-4 and trailer mounted PropakV-3. The RTK position of the trailer was used as truth for analysis of the experimental data. The position of the trailer was estimated using both the encoder and DRTK algorithms for each experiment. The trailer's position was calculated in real-time using the encoder and the position measurement from the DL4-Plus mounted on the robot. This measurement was used by the controller to guide the robot.

\section{IV.B. DRTK vs. Encoder Method}

The result of one experiment (with parameters $l_{r}=0.83 \mathrm{~m}$ and $l_{t}=2.73 \mathrm{~m}$ ) is presented in figures 3 and 4 . The desired path, the estimated trailer position using both methods, and the RTK truth measurement are shown in figure 3 and a zoomed in portion is shown in figure 4 . It can be observed that the DRTK estimate is very close to the true position, while a small bias exists in the encoder estimate.

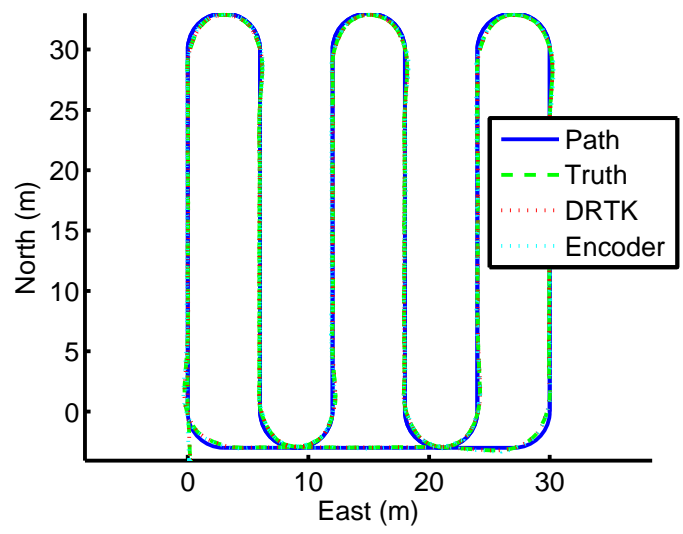

Figure 3. Path followed by Robot and Trailer

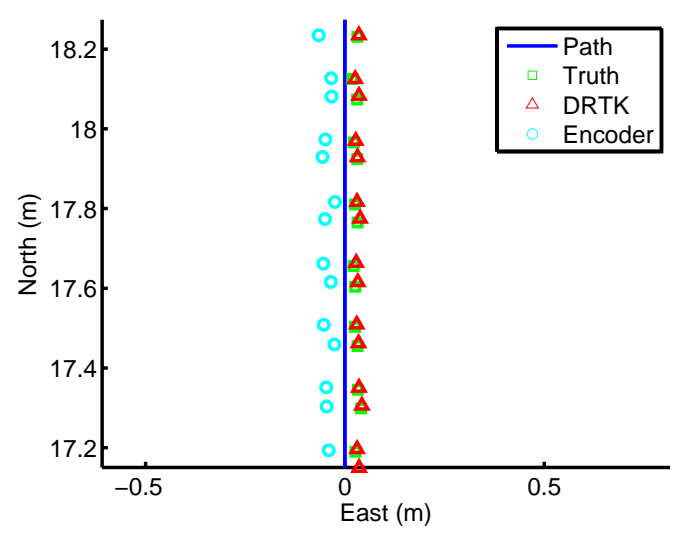

Figure 4. Path followed by Robot and Trailer

The difference between in the trailer's estimated position (determined with both methods) and the truth data (from the second RTK receiver) is shown in figure 5. Approximately 10 minutes of static data was taken at the beginning of each run to allow sufficient time for the DRTK algorithm to converge since the algorithm was being run post-process and therefore the exact convergence time needed was unknown. Convergence was normally much faster than this, however, with convergence times for the experiments ranging from 1 to 4 minutes. The same bias that was seen in figure 4 can also be seen in the plot of total error (figure 5 , particularly during the static data, where it appears that the bias is approximately $6-7 \mathrm{~cm}$. This bias is 
most likely caused by a bias in the encoder measurement. It could possibly be reduced by more carefully aligning the encoder, but alignment can be a difficult and time consuming process. Note: Divelbiss and Wen also came to this conclusion about alignment. ${ }^{9}$ While both methods perform well, the DRTK method has a noticeably smaller error than the encoder method and does not require exact knowledge of the robot geometry or careful encoder calibration.

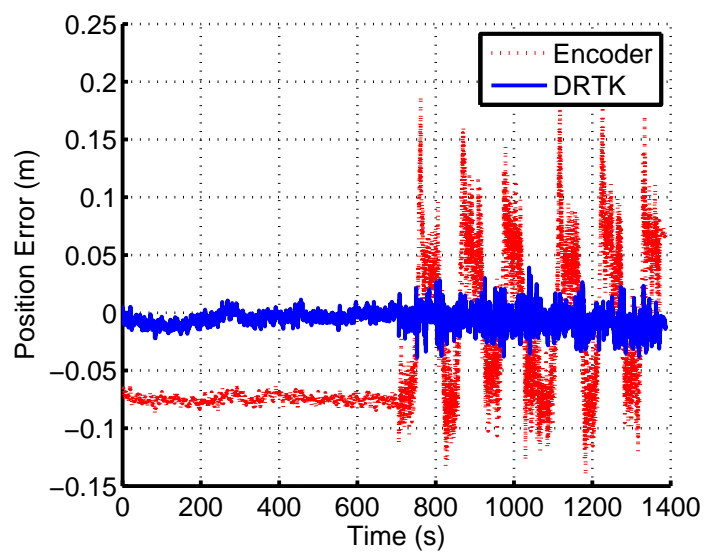

Figure 5. Total Estimation Error

The total estimation error was also broken into lateral and longitudinal components in the vehicle frame. The lateral error is of more importance than the longitudinal error for our application. Longitudinal error will shift the entire suvey area in one direction, but will not affect the area covered within that area. Errors in the lateral position, however, can cause gaps in coverage within the survey area. The lateral and longitudinal error components as functions of time are shown in figure 6 , and a scatter plot of the lateral and longitudinal error is shown in figure 7 . From figure 7 it can be seen that he DRTK method is very accurate with the majority of the points having a total error of less than $1 \mathrm{~cm}$, while the errors from the encoder method are on average $5-6 \mathrm{~cm}$ and can be as large as $25 \mathrm{~cm}$.
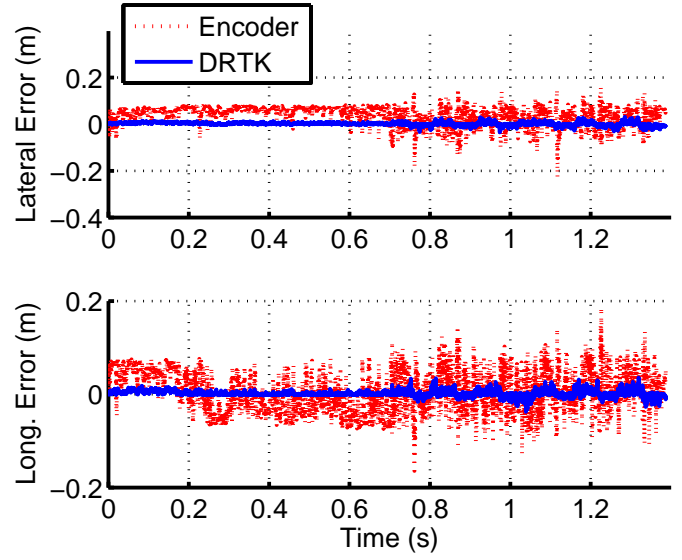

Figure 6. Lateral and Longitudinal Estimation Error

\section{IV.C. Encoder Method with Incorrect Parameters}

For the UXO system described previously, trailer lengths are varied often based on the type and configuration of sensor being towed. Because of this, the trailer length parameter $\left(l_{t}\right)$ changes often. (Note that the tongue length parameter $\left(l_{r}\right)$ is determined by the portion of the tongue that is a part of the robot and does not change.) When a new trailer configuration is used, the length is typically measured in the field using a tape measure and entered into the control software. This process is prone to errors which, when they occur, can significantly affect the performance of the system. An error was made while measuring the trailer length 
during one of the experimental runs performed for this paper. The length entered was off by approximately $10 \mathrm{~cm}$. The resulting lateral and longitudinal error are shown in figure 8. Since the trailer length parameter is used to calculate the trailer's position using Eq. 1, the error in the parameter also appears in the trailer's position estimate. While the error is easy to detect when truth data is available, this information is not normally available and the error would result in inaccurate path following and as a result an inaccurate map of the area being surveyed. The DRTK method, however, is does not require the trailer length parameter and still provides an accurate measurement of the trailer's position.

\section{Effects of Trailer Length}

The effect of changes in the trailer length was examined for both estimation methods. The error in the DRTK measurement is expected to be constant for reasonable trailer lengths. The error from the encoder method, however, should increase with trailer length. The encoder method is sensitive to errors in both the hitch angle $\varphi$ and the robot's orientation $\psi_{r}$. Both have the effect of "swinging" the trailer estimate to one side or the other of the actual position. The sensitivity to these errors is proportional to the hitch and trailer lengths $l_{r}$ and $l_{t}$. Consider a small error $\epsilon$ in the hitch angle measurement as shown in figure 9 . The lateral and longitudinal errors ( $y_{e r r}$ and $x_{e r r}$ respectively) in the estimate of the trailer's position as a result of the error in the hitch angle $(\epsilon)$ are given by:

$$
\begin{aligned}
& x_{e r r}=l_{t}(1-\cos (\epsilon)) \\
& y_{\text {err }}=l_{t} \sin (\epsilon)
\end{aligned}
$$

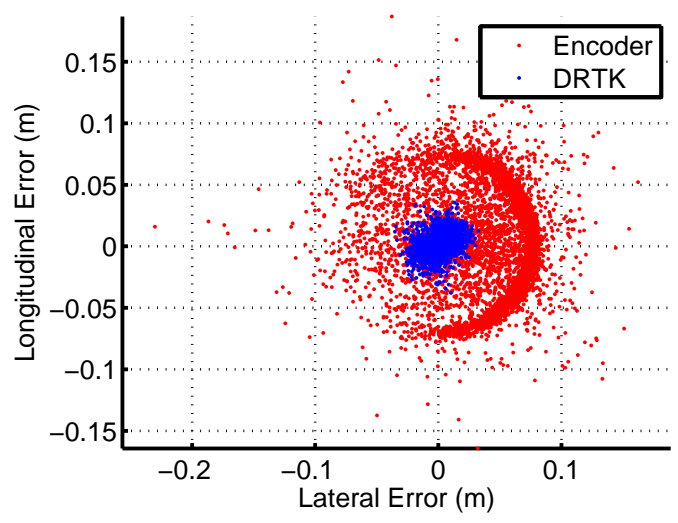

Figure 7. Lateral and Longitudinal Estimation Error Scatter Plot

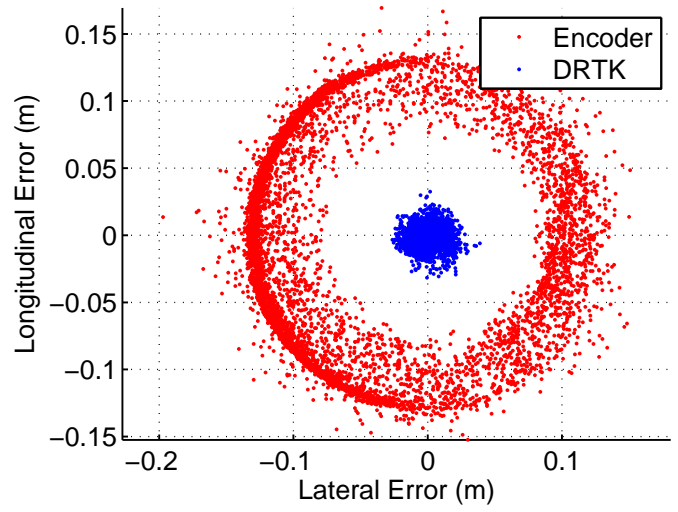

Figure 8. Effect of Errors in Trailer Length Parameter 
Errors in the robot's heading have a similar effect on longitudinal and lateral position with the resulting errors given by:

$$
\begin{aligned}
& x_{\text {err }}=\left(l_{r}+l_{t}\right)(1-\cos (\epsilon)) \\
& y_{\text {err }}=\left(l_{r}+l_{t}\right) \sin (\epsilon)
\end{aligned}
$$

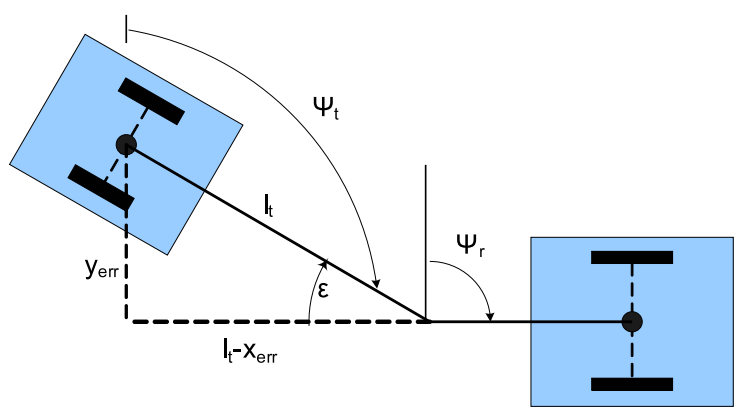

Figure 9. Effect of Hitch Angle Error on Trailer Position

The above equations show that the position error caused by an error in the measurement of the hitch angle are scaled by the trailer parameters $\left(l_{r}\right.$ and $\left.l_{t}\right)$. As a result the longer the trailer is, the more significant errors in the hitch angle measurement are. To validate the above calculations, the experiment described in Sec. IV was repeated for two additional trailer lengths $\left(l_{t}=0.91 \mathrm{~m}\right.$ and $\left.l_{t}=1.83 \mathrm{~m}\right)$. The total, lateral, and longitudinal errors were calculated for both the DRTK and encoder methods for each trailer length. The mean $\bar{x}$ and standard deviation $\sigma_{x}$ were calculated for each error and the results are given in Table 1 for the DRTK method and in Table 2 for the encoder method. A plot showing the total RMS errors as functions of trailer length for the two methods is shown in figure 10. As expected, the errors using the DRTK method stayed essentially constant over the runs, while the errors from the encoder method increased with trailer length.

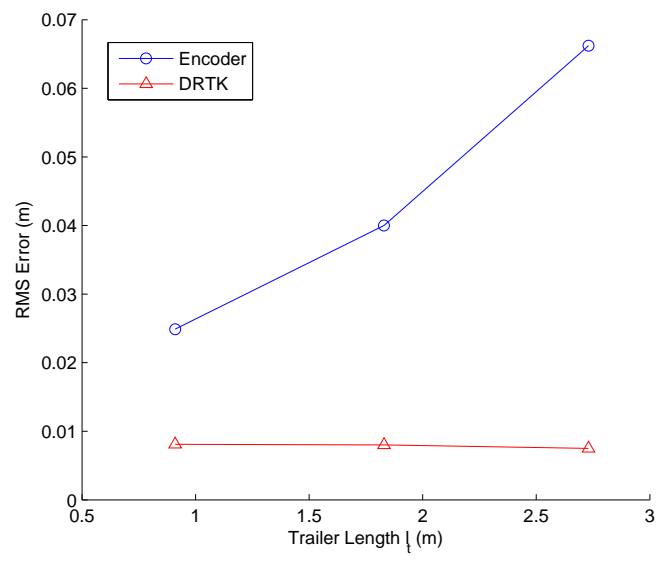

Figure 10. Error vs. Trailer Length

Table 1. Effect of Trailer Length on Errors (DRTK)

\begin{tabular}{c|c|c|c|c|c|c}
\hline Length $(\mathrm{m})$ & \multicolumn{2}{|c|}{ Total $(\mathrm{cm})$} & \multicolumn{2}{c|}{ Lat $(\mathrm{cm})$} & \multicolumn{2}{c}{ Long $(\mathrm{cm})$} \\
\hline$l_{t}$ & $\bar{x}$ & $\sigma_{x}$ & $\bar{x}$ & $\sigma_{x}$ & $\bar{x}$ & $\sigma_{x}$ \\
\hline 0.91 & -0.20 & 1.04 & 0.019 & 0.72 & 0.068 & 0.78 \\
1.83 & -0.13 & 0.85 & 0.13 & 0.88 & 0.10 & 0.97 \\
2.73 & -0.54 & 0.79 & 0.19 & 0.70 & 0.18 & 0.60 \\
\hline
\end{tabular}


Table 2. Effect of Trailer Lenght on Errors (Encoder)

\begin{tabular}{c|c|c|c|c|c|c}
\hline Length $(\mathrm{m})$ & \multicolumn{2}{|c|}{ Total $(\mathrm{cm})$} & \multicolumn{2}{c|}{ Lat $(\mathrm{cm})$} & \multicolumn{2}{c}{ Long $(\mathrm{cm})$} \\
\hline$l_{t}$ & $\bar{x}$ & $\sigma_{x}$ & $\bar{x}$ & $\sigma_{x}$ & $\bar{x}$ & $\sigma_{x}$ \\
\hline 0.91 & 0.23 & 2.98 & 0.65 & 1.97 & -0.34 & 2.12 \\
1.83 & 0.35 & 3.56 & 0.72 & 3.21 & -0.42 & 3.67 \\
2.73 & -0.25 & 2.21 & -0.18 & 1.61 & 0.10 & 1.52 \\
\hline
\end{tabular}

\section{Conclusions}

In this paper, a dynamic base real time kinematic (DRTK) GPS algorithm has been presented. It has been shown to accurately estimate short baselines to within a few centimeters. An application to a robottrailer system was described. It was shown that the DRTK algorithm presented could be used to accurately determine the trailer's position relative to the robot. This method of trailer position determination was experimentally compared to a method that uses an optical encoder to measure the angle between the robot and trailer.

The experimental results showed that the DRTK algorithm could provide a more accurate measurement of the trailer's position than the method using an encoder in areas where GPS coverage is sufficient. The degree of improvement over the encoder method is directly related to the length of the trailer. The DRTK method also eliminates the need for exact knowledge of the system's geometry, removing a possible source of human error from the system.

\section{Acknowledgements}

The authors express thanks for support from the U.S. Army Corps of Engineers and the Environmental Security Technology Certification Program (ESTCP).

\section{References}

${ }^{1} \mathrm{M}$. Nakamura and S. Yuta, "Trajectory control of trailer type mobilie robots," in IEEE/RSJ International Conference on Intelligent Robots and Systems, July 1993.

${ }^{2}$ M. Park and W. Chung, "Control of a mobile robot with passive multiple trailers," in Proceedings of the 2004 IEEE International Conference on Robotics and Automation, New Orleans, LA, April 2004.

${ }^{3}$ S. Sekhavat, F. Lamiraux, J. P. Laumond, G. Bauzil, and A. Ferrand, "Motion planning and control for Hilare pulling a trailer: experimental issues," in Proceedings of the 1997 IEEE International Conference on Robotics and Automation, Albuquerque, NM, April 1997, pp. 3306-3311.

${ }^{4}$ S. De Luca, Oriolo, Robot Motion Planning and Control. Spring, 1998, ch. Feedback Control of a Nonholonomic Car-like Robot, pp. 343-427.

${ }^{5}$ D. W. Hodo, "Development of an autonomous mobile robot-trailer system for UXO detection," Master's thesis, Auburn University, Auburn, AL, August 2007.

${ }^{6}$ S. J. Comstock, "Development of a low-latency, high data rate, differential gps relative positioning system for uav formation flight control," Ph.D. dissertation, Air Force Institute of Technology, 2006.

${ }^{7}$ S. C. Felter and N. E. Wu, "A relative navigation system for formation flight," IEEE Transactions on Aerospace and Electronic Systems, vol. 33, pp. 958-967, 1996.

${ }^{8}$ T. Ford, M. Hardesty, and M. Bobye, "Helicopter ship board landing system," in ION GNSS 18th International Technical Meeting of the Satellite Division, 2005.

${ }^{9}$ A. Divelbiss and J. Wen, "Trajectory tracking control of a car-trailer system," Control Systems Technology, IEEE Transactions on, vol. 5, no. 3, pp. 269-278, May 1997.

${ }^{10}$ F. Lamiraux, S. Sekhavat, and J.-P. Laumond, "Motion planning and control for hilare pulling a trailer," Robotics and Automation, IEEE Transactions on, vol. 15, no. 4, pp. 640-652, Aug. 1999.

${ }^{11}$ U. Larsson, C. Zell, K. Hyyppa, and A. Wernersson, "Navigating an articulated vehicle and reversing with a trailer," in Porceedings of the 1994 International Conference on Robotics and Automation, vol. 3, May 1994, pp. 2398-2404.

${ }^{12}$ R. M'Closkey and R. Murray, "Experiments in exponential stabilization of a mobile robot towing a trailer," in American Control Conference, 1994, vol. 1, 29 June-1 July 1994, pp. 988-993vol.1.

${ }^{13}$ D. W. Hodo, J. Y. Hung, D. M. Bevly, and D. S. Millhouse, "Analysis of trailer position error in an autonomous robottrailer system with sensor noise," in Proc. IEEE International Symposium on Industrial Electronics ISIE 2007, 2007, pp. $2107-2112$. 
${ }^{14}$ D. W. Hodo, J. Y. Hung, D. M. Bevly, and S. Millhouse, "Effects of sensor placement and errors on path following control of a mobile robot-trailer system," in Proc. American Control Conference ACC '07, 2007, pp. 2165-2170.

${ }^{15}$ P. Misra and P. Enge, Global Positioning System: Signals, Measurements, and Performance. Ganga-Jumana Press, 2006.

${ }^{16}$ B. Hofmann-Wellenhof, Global Positioning System : Theory and Practice. Springer-Verlag, 1997.

${ }^{17}$ D. Lawrence, R. Langley, D. Kim, F.-C. Chan, and B. Pervan, "Decorrelation of troposphere across short baselines," in Position, Location, and Navigation Symposium, 2006 IEEE/ION, 2006.

${ }^{18}$ A. Gelb, Applied Optimal Estimation. The M.I.T. Press, 1974.

${ }^{19}$ E. D. Kaplan and C. J. Hegarty, Understanding GPS: Principles and Applications. Artech House, 2006.

${ }^{20} \mathrm{P}$. de Jonge and C. Tiberius, "The lambda method for integer ambiguity estimation: Implementation aspects," Delft Geodetic Computing Centre, Tech. Rep., August 1996.

${ }^{21}$ P. Joosten and C. Tiberius, "Lambda: Faqs," GPS Solutions, vol. 6, pp. 109-114, 2002.

${ }^{22}$ P. Joosten, "The lambda-method: Matlab implementation," Mathematical Geodesy and Positioning, Delft Universtiy of Technology, Tech. Rep., 2001.

${ }^{23}$ P. E. Henderson, "Development and testing of a multiple filter approach for precise dgps positioning and carrier phase ambiguity resolution," Ph.D. dissertation, Air Force Institute of Technology, 2001. 